\title{
Contact of Flexible Elastic Belt with Two Pulleys
}

A.K. Belyaev, V.V. Eliseev, H. Irschik and E.A. Oborin

\begin{abstract}
The drive belt set on two pulleys is considered as a nonlinear elastic rod deforming in plane. The modern equations of the nonlinear theory of rods are used. The static frictionless contact problem for the rod is derived. The nonlinear boundary value problems for the ordinary differential equations are solved by the finite differences method and by the shooting method by means of computer mathematics. The belt shape and the stresses are determined in the nonlinear formulation which delivers the contact reaction and the contact area. The developed method allows performing calculations for any set of geometrical and stiffness parameters.
\end{abstract}

Key words: belt drive, nonlinear elastic rod, contact problem, boundary value problem, computer mathematics

\section{Introduction}

The technical calculations of the belt drives are usually based on the ideas of an inextensible string and the Eulerian formula. However modelling of high loaded drives requires taking into account elastic deformations of the belt. In the previous works $[4,5,11]$ the model of an extensible string is used, and the contour motion with the constant trajectory is explored. The model of a rod with (at least) bending stiffness is needed because the friction force loading does not act on the belt axis.

A number of works consider the significant effect of bending stiffness on the belt drive dynamics $[1,7,8]$. In finite-element modelling $[2,9,10]$, the penalty formula-

\footnotetext{
A.K. Belyaev

Institute for Problems in Mechanical Engineering, Russian Academy of Sciences, Saint-Petersburg

V.V. Eliseev

Peter the Great Saint-Petersburg Polytechnic University, Saint-Petersburg, Russia

H. Irschik · E.A. Oborin

Johannes Kepler University Linz, Austria, e-mail: evgenii.oborin@jku.at
} 
Fig. 1 Contact of straight beam and cylinder

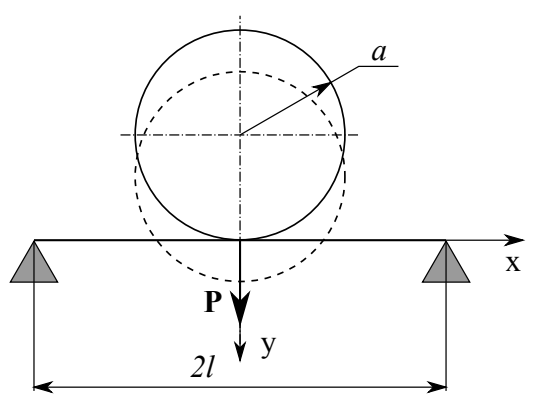

tions are used for obtaining the conditions in the contact area. In the works [10, 12] the concentration of reactions at the contact area boundaries is noted. However accurate solutions even for the static problem with the large deformations inherent to real drives have not been developed. For large deformation problems the nonlinear theory of rods is necessary. The corresponding theory of rods [3] and computer mathematics allow us to formulate and solve difficult nonlinear problems.

The goal of this work is an application of the nonlinear elastic rod theory in the contact problem of fitting the belt as a ring to the frictionless pulleys in order to find strains and forces, reactions and their localization $[6,13]$. Before starting we consider the linear model (see Fig. 1) to explain the localization of the reactions.

In this problem a stiff cylinder with the radius $a$ is pressed against the centre of a simply supported beam with the increasing force $P$. At first the force is concentrated at the contact point, and the deflection of the beam is $\varepsilon=P l^{3} A / 6$. Here the bending compliance is $A$, the length of the beam is $2 l$. The curvature of the beam at the contact point becomes $A M=A P l / 2$. When it reaches the value $1 / a$, i.e. $P=P^{*}=$ $2 / A l a$, the contact zone expands. The displacement of the cylinder points is

$$
v(x)=\varepsilon-a+\sqrt{a^{2}-x^{2}}=\varepsilon-\frac{x^{2}}{2 a}-\frac{x^{4}}{8 a^{3}}+\ldots
$$

Assuming the full contact (when the points of the beam lie on the cylinder), we obtain the contact pressure $p(x)=A^{-1} v^{I V}$. Since $p<0$ the full contact is not present, and the contact area is concentrated at the ends of the interval $\left(x= \pm s_{1}\right)$. The value $s_{1}$ is determined by matching the solutions at the different segments. The dependence $P(\varepsilon)$ becomes nonlinear. Below in Sect. 3 we will use this result to formulate the nonlinear contact problem.

\section{Nonlinear classical theory of rods deforming in plane}

Elastic rods may be considered as Cosserat material lines, whose particles have the translational and rotational degrees of freedom [3]. In Fig. 2 the rod is shown in two configurations: before and after the deformation. 
The rod axis is defined by the dependence of the position vector $\mathbf{r}(s)$ on the material coordinate. A triple of unit vectors $\mathbf{e}_{k}(s)$ is connected with every particle to establish an angular orientation. In Fig. 2 index zero indicates the values before deformation. We restrict ourselves to the model without extension, then the variable $s$ is the arc coordinate in both configurations.

The external loads are the force $\mathbf{q}$ and the moment $\mathbf{m}$ distributed per unit length. The internal interaction is defined by the force $\mathbf{Q}$ and the moment $\mathbf{M}$, whose signs depend on the direction of $s$. The full system of equations involves equations of balance of forces and moments, definitions of strains and elasticity relations [3].

In this work we consider the plane problem of the belt fitted on two pulleys. Thus vectors $\mathbf{r}, \mathbf{Q}, \mathbf{e}_{1}, \mathbf{e}_{2}$ lie in the plane of the drawing. The unit vectors $\mathbf{e}_{10}, \mathbf{e}_{20}$ are rotated with regard to $\mathbf{i}, \mathbf{j}$ by an angle $\alpha(s)$ (around the z-axis). After the deformation we have $\mathbf{e}_{1}, \mathbf{e}_{2}$ and the angle $\varphi=\alpha+\theta$. In the general theory a rotation tensor $\mathbf{P}=\mathbf{e}_{i} \mathbf{e}_{i 0}$ is introduced, but in the plane problem just one angle $\theta$ is sufficient. The moment vector $\mathbf{M}=M \mathbf{k} ; \mathbf{k}=\mathbf{e}_{3}=\mathbf{e}_{30}$ is directed along the $\mathrm{z}$-axis. The vector of bending strain is $\theta^{\prime} \mathbf{k}$, where prime indicates differentiation with respect to $s$.

The equations of the full system [3] are simplified:

$$
\mathbf{Q}^{\prime}=-\mathbf{q}, M^{\prime}=-\mathbf{k} \cdot \mathbf{r}^{\prime} \times \mathbf{Q}=-x^{\prime} Q_{y}+y^{\prime} Q_{x}, \theta^{\prime}=A M, \mathbf{r}^{\prime}=\mathbf{P} \cdot \mathbf{r}_{0}^{\prime} .
$$

$A$ is the bending compliance. Since $\mathbf{r}_{0}^{\prime}=\mathbf{e}_{10}$, and thus $\mathbf{P} \cdot \mathbf{e}_{k 0}=\mathbf{e}_{k}$, we can write

$$
\mathbf{r}^{\prime}=\mathbf{e}_{1} \Rightarrow x^{\prime}=\cos \varphi, y^{\prime}=\sin \varphi
$$

\section{Contact problem formulation}

The scheme of the belt on two equal pulleys (with the radius $a$ ) is depicted in Fig. 3 . Motivated by the above linear model (see Sect. 1), we begin the analysis under the assumption of the contact at discrete points. The half of the center distance before the deformation equals $d=R-a$ and then it is increasing by given value $\varepsilon$. We aim

Fig. 2 Initial (dashed line) and deformed (solid line) rod configurations: position vectors, local basis, angles

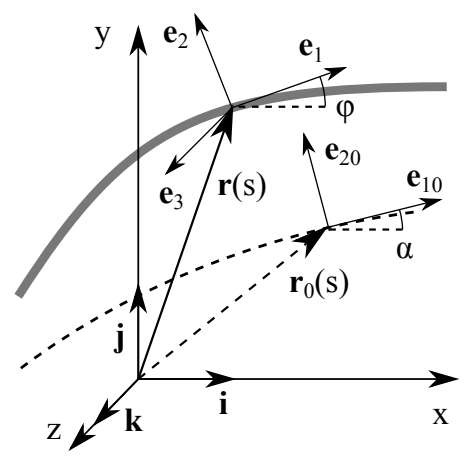


at computing the deformation and the contact forces to obtain a given kinematical loading $\varepsilon$. The problem has two axes of symmetry, thus the consideration of first quarter $0<s<L=\pi R / 2$ is sufficient. The load $\mathbf{q}$ is just the reaction force from the pulley $\mathbf{N}$ that is concentrated in the contact point with the unknown coordinate $s_{1}$ : $\mathbf{q}=\mathbf{N} \delta\left(s-s_{1}\right)$ (here $\delta$ is the Dirac delta-function). Then the equations of balance of forces and moments in (2) are integrated as:

$$
\begin{aligned}
\mathbf{Q} & =-\mathbf{N} h\left(s-s_{1}\right)+\mathbf{Q}_{0} ; \\
M & =\mathbf{k} \cdot\left[\left(\mathbf{r}-\mathbf{r}_{1}\right) \times \mathbf{N} h\left(s-s_{1}\right)-\mathbf{r} \times \mathbf{Q}_{0}\right]+M_{*} .
\end{aligned}
$$

Here $h(\ldots)$ is the unit step function (Heaviside), $\mathbf{Q}_{0}, M_{*}$ are the vector and the scalar constants of integration. In the absence of friction the contact force $\mathbf{N}$ is directed perpendicular to the pulley. We denote $\gamma$ as the angle of the force to the $\mathrm{x}$-axis: $\mathbf{N}=N(\mathbf{i} \cos \gamma+\mathbf{j} \sin \gamma)$. At the contact point we have $\varphi=\gamma-\pi / 2$. At the ends of the interval, transverse forces equal to zero: $\mathbf{Q}(0)=\mathbf{Q}_{0}=Q_{0} \mathbf{i}, \mathbf{Q}(L)=-Q_{L} \mathbf{j}$. From the equation of balance of forces for the quarter of the rod, it follows:

$$
\left.\mathbf{Q}\right|_{0} ^{L}+\mathbf{N}=0 ; N \cos \gamma=Q_{0} \equiv P / 2, Q_{L}=N \sin \gamma
$$

The tension $Q_{0}$ at the top point equals to the half of the force $P$ moving the pulleys apart. The tension in the right point $Q_{L}=P \tan \gamma / 2$. The bending moment

$$
M=N\left[\left(x-x_{1}\right) \sin \gamma-\left(y-y_{1}\right) \cos \gamma\right] h\left(s-s_{1}\right)+y Q_{0}+M_{*}
$$

with the contact point $\mathbf{r}_{1}=x_{1} \mathbf{i}+y_{1} \mathbf{j}=(d+\varepsilon+a \cos \gamma) \mathbf{i}+a \sin \gamma \mathbf{j}$.

We note that in the considered model without axial extension the coordinate $s$ remains the arc coordinate also after the deformation. Thus, $\alpha=-s / R, \varphi=\theta-s / R$. The derived system of ordinary differential equations (ODE) can be solved partly due to the existence of the first integrals. Within both segments of the interval $(0, L)$, there is $\mathbf{Q}=$ const, and then it follows:

Fig. 3 Fitting of the ring on the pulleys. Here solid circles are the pulleys, dashed circle is the initial belt configuration and solid line is the deformed configuration

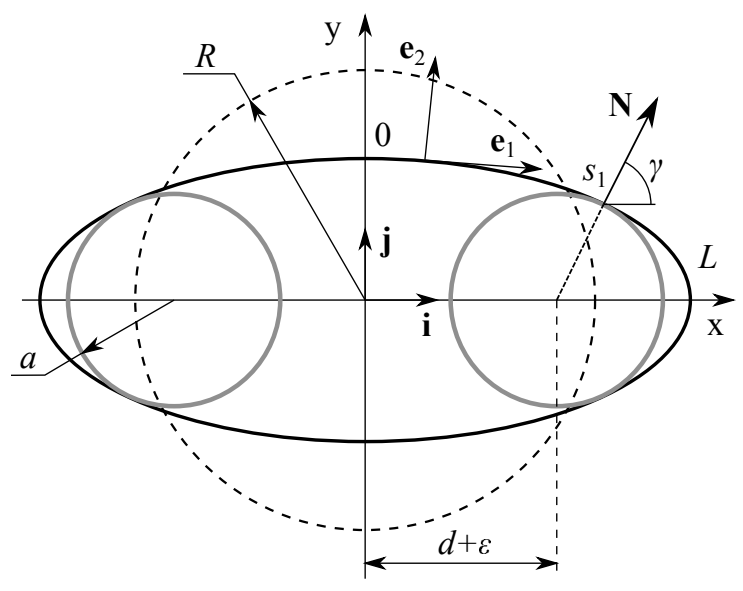




$$
A^{-1} \theta^{\prime \prime}=y^{\prime} Q_{x}-x^{\prime} Q_{y}=Q_{x} \cos (s / R-\theta)-Q_{y} \sin (s / R-\theta) .
$$

Introducing the variable $\varphi(s)$ as above, we obtain the equation:

$$
A^{-1} \varphi^{\prime \prime}-Q_{x} \cos \varphi-Q_{y} \sin \varphi=0 .
$$

It is integrated in terms of elliptic functions as in the classical problems [3]:

$$
\begin{gathered}
A^{-1} \varphi^{\prime 2} / 2-Q_{x} \sin \varphi+Q_{y} \cos \varphi=c_{1}=\text { const } \\
\int \frac{\mathrm{d} \varphi}{\sqrt{c_{1}+Q_{x} \sin \varphi-Q_{y} \cos \varphi}}=s \sqrt{2 A}+c_{2} .
\end{gathered}
$$

However it is difficult to determine $c_{1}, c_{2}$ that are different at both segments.

We suggest another approach, which is more advantageous. The unknown constants of the problem are $s_{1}, \gamma, Q_{0}, M_{*}$. The unknown functions are $\theta(s), x(s), y(s)$. Considering the constants as functions, we equate their derivatives to zero (see e.g. this well-known method in [8]). Adding the expressions $\theta^{\prime}, x^{\prime}, y^{\prime}$, we arrive to the ODE system of seventh order. The boundary conditions are:

$$
\begin{aligned}
& s=0: \theta=0, x=0 ; s=L: \theta=0, y=0 \\
& s=s_{1}: \theta=\theta_{1}=s_{1} / R+\gamma-\pi / 2, x=x_{1}=d+\varepsilon+a \cos \gamma, y=y_{1}=a \sin \gamma
\end{aligned}
$$

Four conditions at the ends $s=0, L$ are not enough for the seventh order system. Conditions at the contact point should be taken into account.

The formulated problem with the condition in the internal point is not usual and is not solved in particular by the shooting method. We can transform it to the tenth order system at the interval $[0,1]$ with the appropriated number of conditions. Instead of the coordinate $s$ we introduce a new variable $\xi \in[0,1]$ by setting

$$
s=\left\{\begin{array}{c}
\xi s_{1}, s<s_{1}, \\
L-\xi\left(L-s_{1}\right), s>s_{1} .
\end{array}\right.
$$

The relation between the derivatives is $(\ldots)=m(s)(\ldots)^{\prime}$ (the point means differentiation with respect to $\xi$ ); here $m=s_{1}$ at the first interval and $m=s_{1}-L$ at the second one. We shall consider the solutions at this two intervals as two different unknowns.

\section{The solution using computer mathematics}

Constructing a column of the unknowns $Y(\xi)$ with 10 elements, we have:

$$
Y=\left(s_{1} \gamma Q_{0} M_{*} \theta^{(1)} x^{(1)} y^{(1)} \theta^{(2)} x^{(2)} y^{(2)}\right)^{T},
$$

then we arrive to the following system of ODEs:

$$
\dot{Y}=F(\xi, Y)=\left(\begin{array}{llllllllllll}
0 & 0 & 0 & 0 & F_{4} & F_{5} & F_{6} & F_{7} & F_{8} & F_{9}
\end{array}\right)^{T},
$$




$$
F_{4}=m A M, F_{5}=m \cos \left(\theta^{(1)}-s / R\right), F_{6}=m \sin \left(\theta^{(1)}-s / R\right) .
$$

Components $F_{7}, F_{8}, F_{9}$ differ by the expressions $m, M, \theta$ at the segments (6). We should add to the system (see the end of Sect. 3 and (11)) the boundary conditions (9). This boundary value problem (BVP) is solved by means of computer mathematics, namely by the finite difference method using Wolfram Mathematica and by the shooting method using Mathcad. The results of both methods are equal.

In the example calculation the radius of the ring is $R=0.55 \mathrm{~m}$, the radius of the pulleys is $a=0.15 \mathrm{~m}$, the half of center distance is $d=0.4 \mathrm{~m}$. The Young's modulus is $E=0.1 \mathrm{GPa}$, and the section is a square with the side equal $0.01 \mathrm{~m}$. In a series of calculations the increment $\varepsilon$ of distance $d$ is increased from zero to the limiting value due to the inextensibility. Figure 4 corresponds to the displacement $\varepsilon=0.228 \mathrm{~m}$. Herewith the angle $\gamma=0.713$, the coordinate $s_{1}=0.758 \mathrm{~m}$ (with the length $L=0.864 \mathrm{~m}$ ), and the force $P=2 Q_{0}=19.6 \mathrm{~N}$.

However the calculations did not reveal such pronounced gaps of the belt from the pulley as in Fig. 3. The configuration of the belt differs from the pulley circle and intersects it. We can assume full contact along with a concentrated contact reaction. Its value is $N=Q_{0} / \cos \gamma=13.0 \mathrm{~N}$. The difference between Fig. 3 and Fig. 4 requires further study presented in what follows.

\section{Version with full contact of belt and pulleys}

Here we assume the full (distributed) contact of the belt on the pulley when $s>s_{1}$. Projecting eq. (2) on the tangent (index 1) and the normal (index 2), we obtain:

$$
Q_{1}^{\prime}+a^{-1} Q_{2}+q_{1}=0, Q_{2}^{\prime}-a^{-1} Q_{1}+q_{2}=0
$$

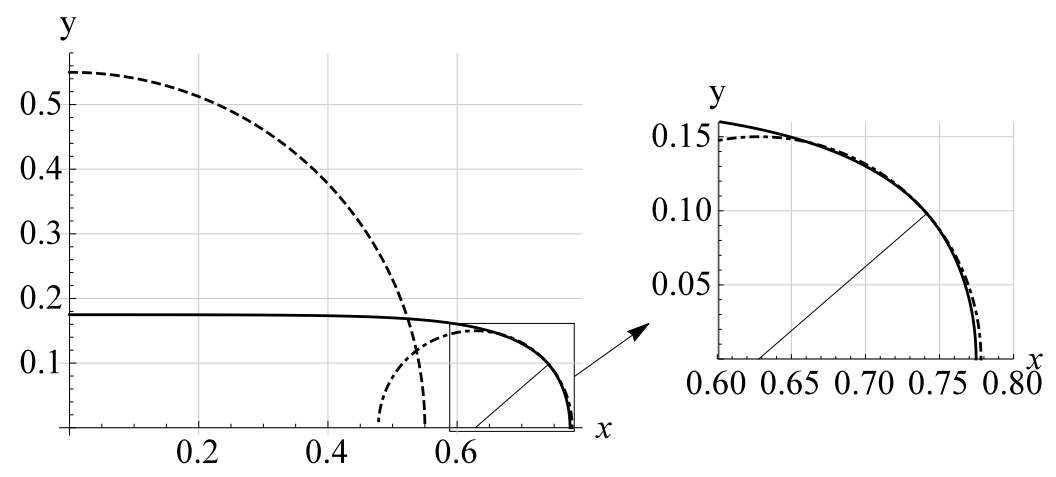

Fig. 4 Belt and pulley: overall picture and intersection. Dashed circle is the initial belt, dash-dot line is the pulley, solid line is the deformed belt, and thin line depicts the contact point 
(taking into account the equalities $\mathbf{e}_{1}^{\prime}=-a^{-1} \mathbf{e}_{2}, \mathbf{e}_{2}^{\prime}=a^{-1} \mathbf{e}_{1}$ ). But $q_{1}=0$, because friction is absent. Transverse force $Q_{2}$ vanishes since the moment is constant

$$
\varphi=a^{-1}(L-s)-\pi / 2, M=A^{-1}\left(R^{-1}-a^{-1}\right), Q_{2}=-M^{\prime}=0 .
$$

Then from (12) we obtain $Q_{1}=$ const $=a q_{2}$, the tension and the contact pressure are constant. Adding obvious expressions $x, y$ at the pulley circle, we obtain all the set of formulas at the contact area having the unknown pair of constants $s_{1}, q_{2}$.

Let us turn to the free interval $s<s_{1}$. Here the above shown relations are applicable: $\mathbf{Q}=Q_{0} \mathbf{i}, Q_{0}=P / 2, M=y Q_{0}+M_{*}$. The last helps us to derive the relation for $M_{*}$. At the point $s=s_{1}$ the moment is continuous and $y=a \sin \gamma$ with angle $\gamma \equiv\left(L-s_{1}\right) / a$. Using (13) we write

$$
M_{*}=-a \sin \left(a^{-1}\left(L-s_{1}\right)\right) Q_{0}+A^{-1}\left(R^{-1}-a^{-1}\right) .
$$

As in (10) we introduce the coordinate $\xi$. Thus we obtain a BVP for the ODE system of fifth order (boundary conditions are almost the same as in (9) ):

$$
\begin{aligned}
\dot{s}_{1} & =0, \dot{Q}_{0}=0, \dot{\theta}=s_{1} A M, \dot{x}=s_{1} \cos \left(\theta-\xi_{s_{1}} / R\right), \dot{y}=s_{1} \sin \left(\theta-\xi_{s_{1}} / R\right) ; \\
\xi & =0: \theta=0, x=0 ; \xi=1: \theta=\theta_{1}, x=x_{1}, y=y_{1} .
\end{aligned}
$$

Solving problem (15) by means of computer mathematics, for $\varepsilon=0.228 \mathrm{~m}$ we obtain $s_{1}=0.672 \mathrm{~m}, Q_{0}=43.8 \mathrm{~N}, \gamma=1.28$.

Friction is absent, therefore at the point $s_{1}$ the tension is continuous: $Q_{0} \sin \gamma=$ $Q_{1}$, so $q_{2}=P \sin \gamma / 2 a=280 \mathrm{~N} / \mathrm{m}$. But the concentrated reaction $N=P \cos \gamma / 2=$ $12.5 \mathrm{~N}$ arises. This value is less than the same one for the point contact problem.

Figure 5 displays the dependence of the angle $\gamma(\varepsilon)$ and the force $P(\varepsilon)$ that moves apart the pulleys. Initially the contact is at one point: $\gamma=0$ until the value $\varepsilon \approx$ $0.19 \mathrm{~m}$. This is consisent with the linear model in Fig. 1. But the dependence of force $P(\varepsilon)$ (Fig. 5) is nonlinear right away, because here we deal with geometric nonlinearity. Quantitative differences are observable at large values of $\varepsilon$.
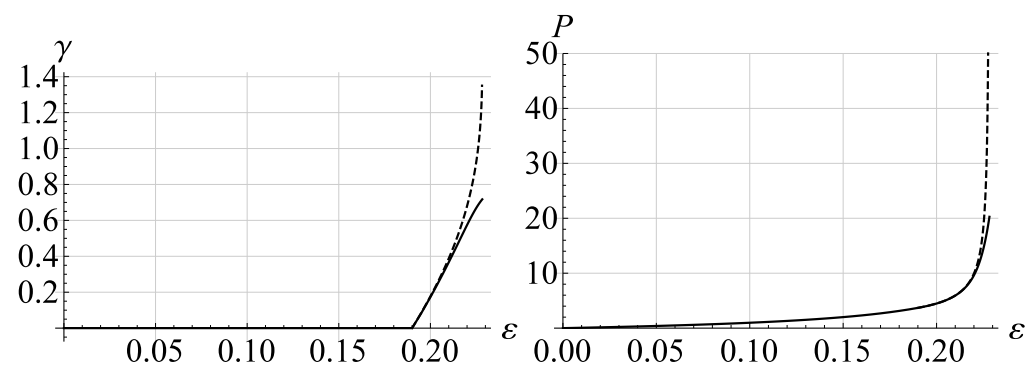

Fig. 5 Numerical results: a angle of reaction inclination; b force moving apart pulleys. Here dashed line depicts the full contact, and solid line depicts the point contact 


\section{Conclusion}

We formulated and solved two contact problems for plane rods for modelling the process of fitting of the belt on the pulleys. Difficulties of solving the nonlinear boundary value problems of tenth and fifth order were overcome by means of computer mathematics. We considered two formulations: the point contact and the full contact, the latter being the preferable one. The strains in both formulations nearly coincide, but the reactions of the pulleys are different. We determined the forms of belt, the stress states, the contact pressures and their localization. The developed method allows us performing multivariant calculations easily.

This research is carried out in the framework of the joint project of the Russian Foundation for Basic Research (grant No. 14-51-15001) and the Austrian Science Fund (FWF, grant No. I 2093 International Project).

\section{References}

1. Ding, H., Li, D.P.: Static and dynamic behaviors of belt-drive dynamic systems with a one-way clutch. Nonlinear Dyn. 78(2), 1553-1575 (2014). DOI 10.1007/s11071-014-1534-7

2. Dufva, K., Kerkkänen, K., Maqueda, L., Shabana, A.: Nonlinear dynamics of threedimensional belt drives using the finite-element method. Nonlinear Dyn. 48(4), 449-466 (2007). DOI 10.1007/s11071-006-9098-9

3. Eliseev, V.: Mechanics of deformable solid bodies. St.-Peterbg. State Polytech. Univ. Publ. House (2006)

4. Eliseev, V.: A model of elastic thread for transmissions with flexible coupling. Sci. and Tech. Bull. of St.-Peterbg. State Polytech. Univ. 84, 192-195 (2009)

5. Eliseev, V., Vetyukov, Y.: Effects of deformation in the dynamics of belt drive. Acta Mech. 223(8), 1657-1667 (2012). DOI 10.1007/s00707-012-0675-3

6. Galin, L.A.: Contact problems. Springer Neth., Dordrecht (2008). DOI 10.1007/978-1-40209043-1

7. Hwang, S.J., Perkins, N.: High speed stability of coupled band/wheel systems: theory and experiment. J. Sound and Vib. 169(4), 459-483 (1994). DOI 10.1006/jsvi.1994.1029

8. Kong, L., Parker, R.G.: Steady mechanics of belt-pulley systems. J. Appl. Mech. 72(1), 25-34 (2005). DOI 10.1115/1.1827251

9. Sinwel, A., Gerstmayr, J.: Modelling an axially moving beam using the absolute nodal coordinate formulation. In: Proc. 7th Int. Conf. on Eng. Comput. Technol., p. 112. Civil Comp Press, Stirlingshire (2010). DOI 10.4203/ccp.94.112

10. Čepon, G., Boltežar, M.: Dynamics of a belt-drive system using a linear complementarity problem for the belt-pulley contact description. J. Sound and Vib. 319(3-5), 1019-1035 (2009). DOI 10.1016/j.jsv.2008.07.005

11. Vetyukov, Y., Eliseev, V., Krommer, M.: Modeling the dynamics of a flexible belt drive using the equations of a deformable string with discontinuities. IFAC-PapersOnLine 48(1), 604-609 (2015). DOI 10.1016/j.ifacol.2015.05.097

12. Wasfy, T.M., Leamy, M.: Effect of bending stiffness on the dynamic and steady-state responses of belt-drives. In: ASME Des. Eng. Tech. Conf., pp. 217-224 (2002). DOI 10.1115/DETC2002/MECH-34223

13. Wriggers, P., Laursen, T.A.: Computational contact mechanics. Springer, Berlin (2006). DOI 10.1007/978-3-540-32609-0 